\title{
Overview of the \\ 1994 ARPA Human Language Technology Workshop
}

\author{
Clifford J. Weinstein, Chair, Editor \\ MIT Lincoln Laboratory \\ Lexington, MA 02173
}

\section{INTRODUCTION}

This volume presents papers, session summaries, and project summaries from the Second ARPA Human Language Technology Workshop, which was held at the Merrill Lynch Conference Center in Plainsboro, NJ, March 8-11, 1994. The Workshop was actually the seventh in a series of ARPA workshops which began in 1988; the first five were called the DARPA Speech and Natural Language Workshops, and the name was changed to the ARPA Human Language Technology (HLT) Workshop in 1993 to reflect the broadened focus and increasing unification of ARPAsponsored research in spoken and written language. In addition, the " $\mathrm{D}$ " was dropped from DARPA in 1993, reflecting a broadening of its charter. The Proceedings of the seven Workshops, all of which have been published by Morgan-Kaufmann, represent a rich source of information on the rapid progress in spoken and written language technology which has been achieved over the past decade, due in no small measure to the leadership of the ARPA Program Managers.

\section{THE 1994 ARPA HUMAN LANGUAGE TECHNOLOGY WORKSHOP}

As with past workshops, the 1994 HLT Workshop provided a forum where researchers were able to share information about very recent technical progress in a highly interactive setting. The scope included all areas of spoken and written language research under ARPA's HLT program, including speech recognition, speech understanding, text understanding, information retrieval, and machine translation, with an emphasis on topics of particular current interest such as evaluation of language understanding systems, and statistical and learning methods. The majority of the workshop participants receive funding under ARPA's HLT program. Other participants included: government researchers and users of the technology; researchers not funded by ARPA who participate voluntarily in these programs; and selected visitors from both inside and outside the United States. Non-U.S. participation was particularly strong in 1994, with 30 attendees representing 9 countries; many of these non-U.S. attendees participated directly and voluntarily in the various formal evaluations of human language systems. In all, there were 230 attendees at the 1994 Workshop, consisting of approximately 150 from ARPA sites, 30 U.S. government representatives, 30 non-U.S. participants, and 20 non-ARPA attendees from the U.S.

For the first time, this HLT Workshop directly followed an ARPA Spoken Language Technology (SLT) Workshop which was held at the same location on March 6-8. This allowed the detailed reporting and discussion of the latest evaluations of speech recognition and spoken language systems to be held at the SLT Workshop, allowing time for broader coverage of technical topics at the HLT Workshop. It also facilitated the attendance at both Workshops of the non-U.S. participants in these evaluations. The SLT Workshop was chaired by Richard Stem of Carnegie Mellon University and attracted approximately 125 attendees. A separate SLT Workshop Proceedings is being published by Morgan Kaufmann.

\section{HUMAN LANGUAGE TECHNOLOGY PROGRAM OVERVIEW}

George Doddington, ARPA Program Manager for Human Language Technology, began the Workshop with an overview of the program's motivation, mission, theme areas, accomplishments, and future directions. Doddington emphasized the key role of Human Language Technology in providing people with the ability to effectively use the National Information Infrastructure. He stated a three-part Human Language Technology Program Mission, to:

- develop Human Language Technologies of key importance; 
- demonstrate Human Language Technologies in compelling application contexts; and

- transfer Human Language Technologies into productive use.

Doddington emphasized the dual roles of technology R\&D and technology transfer in serving this mission. In the ARPA HLT Program, R\&D progress is driven by establishing formal technical challenge tasks in the theme areas including speech recognition, speech understanding, document retrieval, information extraction from text, and machine translation, and by providing infrastructure support including corpus development and regular, formal evaluations of the technology. Technology transfer is driven by identifying critical needs and technology transfer champions, and supporting the transfer with focussed R\&D. Doddington summarized and highlighted recent progress in: spoken language understanding in the Air Travel Information System (ATIS) task; large-vocabulary continuous speech recognition; document detection; and machine translation. With regard to new technical directions, he highlighted the current investigation of a taskindependent evaluation of understanding, referred to as Semantic Evaluation (SemEval), which constituted a major topic of discussion later in the Workshop.

\section{HIGHLIGHTS OF THE WORKSHOP SESSIONS}

The Workshop was comprised of 14 sessions, including a government panel session and a demonstration session. For a good overview of the technical content of the workshop, the reader is encouraged to first read the Session Chairs' summaries which precede the collected papers from each of the sessions. These summaries provide perspective on the research reported as well as outlining the key points in each set of papers.

A few of the highlights of the Workshop included:

- The impressive progress reported by the Linguistic Data Consortium (three papers in Session 1) in collecting and disseminating lexicons, text resources, and speech corpora which are supporting the advancement of Human Language Technology worldwide.

- The new hub and spoke paradigm for largevocabulary continuous speech recognition
(CSR) evaluation, which has successfully balanced common evaluation and diverse research goals.

- The Human Language Evaluation Session, which included many diverse views on the evaluation of language understanding and a spirited discussion of the Semantic Evaluation (SemEval) approaches currently being explored in the ARPA HLT community.

- A strong Machine Translation Session, including a report on a very substantial recent evaluation which included 19 participants; the fact that all but three of these participants were volunteers not supported by ARPA is indicative of ARPA's world leadership role in this area.

- A Demonstration Session, organized and chaired by Victor Abrash, which included demonstrations of HLT for command and control data access, spoken language translation, access to data sources on the information highway, text retrieval and understanding, and reading education.

- A double-length session on Statistical and Learning Methods, highlighting the continuing progress in corpus-based approaches to text understanding.

- A Government Panel, organized and chaired by Oscar Garcia, which included both U.S. and international views on the directions of Human Language Technology.

- A New Directions Session, highlighted by a presentation by a Canadian astronaut and speech researcher, Julie Payette, on applications of speech recognition in space, and also including two papers describing ways in which speech recognition technology has been applied to the automatic recognition of handwritten text.

\section{ACKNOWLEDGMENTS}

The success of this Workshop was due to the hard work of many people. A special thanks goes to James Glass (MIT-LCS), who served as Workshop Vice-Chair and contributed greatly to all aspects of the Workshop organization. The Workshop Planning Committee was responsible for reviewing and selecting papers and demonstrations for presentation, shaping the overall Workshop program, and setting 
Workshop policies. Members of that committee were: James Allen (U. Rochester), Madeleine Bates (BBN), Michael Cohen (SRI), Oscar Garcia (NSF), Ralph Grishman (NYU), Donna Harman (NIST), Lynette Hirschman (MITRE), Eduard Hovy (ISI), Paul Jacobs (GE), Mitchell Marcus (U. Penn), Mari Ostendorf (BU), Richard Schwartz (BBN), Richard Stern (CMU), the ViceChair, James Glass (MIT-LCS), and myself.

Six of those people (Bates, Garcia, Grishman, Marcus, Ostendorf, and Weinstein) also served on the Workshop Standing Committee, chaired this year by Mitch Marcus, which is responsible for overall organization and continuity of the series of ARPA workshops.

A special acknowledgment also goes to Richard Stern, who chaired the SLT Workshop which preceded this one, and helped make the coordination go very smoothly.

A great deal of credit and a very special thanks goes to Linda Nessman (MIT Lincoln Laboratory) who served as Workshop Administrator, which means everything from email archivist to registrar. She contributed to all aspects of Workshop organization. She handled registration, finance, paper abstracts, and the varied requests and problems of the many attendees. She prepared the notebooks of preliminary papers that were handed out at the Workshop, and collected all the final papers and summaries and assembled this volume in photoready form.

Victoria Palay (MIT-LCS), a past Workshop Administrator, provided very valuable help and guidance in many of the administrative aspects of the Workshop, and particularly in setting up the various databases.

Victor Abrash (SRI) deserves particular thanks for taking complete charge of the demonstration session and handling the many difficult details involved in arranging and coordinating a large number of live demos.

I would like to acknowledge all the Session Chairs, who did an excellent job of keeping the sessions on track and contributed the excellent session summaries contained in these Proceedings. The chairs were: George Miller (Princeton), Xuedong Huang (Microsoft), Lynette Hirschman (MITRE), Eduard Hovy (ISI), Paul Jacobs (GE), Madeleine Bates (BBN), Victor Abrash (SRI), Frederick Jelinek (Johns Hopkins), Oscar Garcia (NSF), Steven Young (Cambridge
U.), Donna Harman (NIST), Richard Schwartz (BBN), and Richard Stern (CMU).

As Human Language Technology Program Manager at ARPA, George Doddington provided overall direction and encouragement to the Workshop planners, and was particularly helpful to me as Workshop Chair. His enthusiasm for the program and his devotion to technical excellence were crucial in making this Workshop a resounding success. 